\title{
ON AN INFINITE-DIMENSIONAL DIFFERENTIAL EQUATION IN VECTOR DISTRIBUTION WITH DISCONTINUOUS REGULAR FUNCTIONS IN A RIGHT HAND SIDE
}

\author{
MICHAEL V. BASIN \\ Institute of Control Science, Moscow, Russia \\ 36-1-135, Matveevskaya ul., Moscow 119517 \\ Russia \\ E-mail:basin@matv.msk.ru
}

(Received April, 1995; Revised September, 1995)

\begin{abstract}
An infinite-dimensional differential equation in vector distribution in a Hilbert space is studied in case of an unbounded operator and discontinuous regular functions in a right-hand side. A unique solution (vibrosolution) is defined for such an equation, and the necessary and sufficient existence conditions for a vibrosolution are proved. An equivalent equation with a measure, which enables us to directly compute jumps of a vibrosolution at discontinuity points of a distribution function, is also obtained. The application of the obtained results to control theory is discussed in the conclusion.
\end{abstract}

Key words: Infinite-Dimensional Equation, Discontinuous Right-hand Side, Distribution.

AMS (MOS) subject classifications: $34 \mathrm{~K} 35$.

\section{Introduction}

This paper studies an infinite-dimensional differential equation in vector distribution, whose right-hand side also contains discontinuous regular (not generalized) functions. It should be noted that a solution to a differential equation in distribution cannot be defined as a conventional solution (using the Lebesgue-Stieltjes integral) owing to multiplication of the distribution by a discontinuous regular function. Thus, the basic problems are to introduce an appropriate solution (vibrosolution), obtain the existence and uniqueness conditions for a vibrosolution, and design an equivalent equation with a measure, which enables us to directly compute jumps of a vibrosolution at discontinuity points of a distribution function.

Infinite-dimensional equations in vector distribution appear, for example, when solving the ellipsoidal guaranteed estimation problem [14] over discontinuous observations [2], or considering infinite-dimensional (solid state) impulsive Lagrangian systems [4]. The definition of a unique vibrosolution to a differential equation is first introduced in the background paper [9] and is shown again in Section 3. Finite-dimensional differential equations in scalar distribution with discontinuous regular functions in right-hand sides are studied in [1]. Finite-dimensional equations in vector distribution are then considered in [3]. This paper generalizes the results ob- 
tained in $[1,3]$ to the case of infinite-dimensional differential equations in vector distribution. The substantiation of existence and uniqueness conditions is based $[7,8]$ on the representation of a solution to a differential equation in a Hilbert space as a Fourier sum of solutions to finitedimensional differential equations.

The paper is organized as follows. The problem statement is given in Section 1. In Section 2 a solution to an infinite-dimensional equation in vector distribution is introduced as a vibrosolution, that is defined as a unique limit. Sections 3 and 4 present the necessary and sufficient existence conditions for a vibrosolution, respectively. By definition, existence of a vibrosolution yields its uniqueness. In Section 5 an equivalent equation with a measure is designed. The application of the obtained results to control theory is discussed in the conclusion.

\section{Problem Statement}

Let us consider an infinite-dimensional differential equation in vector distribution with an unbounded operator in a right-hand side

$$
\dot{x}(t)=A x(t)+f(x, u, t)+\beta(x, u, t) b(x, u, t) \dot{u}(t), \quad x\left(t_{0}\right)=x_{0},
$$

where $x(t) \in H ; A$ is a generator of a strongly continuous semigroup such that $(-A)$ is a strongly positive operator and has a compact inverse operator $A^{-1} ; f(x, u, t) \in H, b(x, u, t) \in L\left(R^{m} \rightarrow H\right)$ are bounded continuous functions defined in the space $H \times R^{m} \times R, L(\mathcal{A} \rightarrow \mathfrak{B})$ is a space of linear continuous operators from a space $\mathcal{A}$ to a space $\mathfrak{B} ; \beta(x, u, t) \in R$ is a scalar piecewise continuous in $x, u, t$ function such that its continuity domain is locally connected; $u(t)=\left(u_{1}(t), \ldots, u_{m}(t)\right) \in$ $R^{m}$ is a vector bounded variation function which is non-decreasing in the following sense: $u\left(t_{2}\right) \geq u\left(t_{1}\right)$ as $t_{2} \geq t_{1}$, if $u_{i}\left(t_{2}\right) \geq u_{i}\left(t_{1}\right)$ for $i=1, \ldots, m$.

Let $S_{t}(\cdot): H \rightarrow H$ be a strongly continuous semigroup generated by an operator $A$, and $D(A) \subset H$ be a definition domain. The following conditions imposed on an initial value and a right-hand side of the equation (1): 1) $\left.S_{t}\left(x_{0}\right) \in D(A), 2\right) S_{t-s}(f(x, u, s)+\beta(x, u, s) b(x, u, s)$ $\dot{w}(s)) \in D(A), s \leq t$, are assumed to hold for any absolutely continuous non-decreasing function $w(s) \in R^{m}$.

\section{Definition of a Solution}

Let us note that a solution to the equation (1) cannot be defined as a conventional solution owing to multiplication of distribution $\dot{u}(t)$ by a discontinuous in $t$ function $b(x(t), u(t), t)$.

If $u(t) \in R^{m}$ is an absolutely continuous function, then an absolutely continuous solution to the equation (1) is defined in the sense of Filippov [6]. Following [6], a function $\kappa(x, u, t)$ is said to be piecewise continuous in a finite domain $G \subset H \times R^{m+1}$ if

1) a domain $G$ consists of a finite number of continuity domains $G_{i}, i=1, \ldots, m$, with

boundaries $\Gamma_{i}$,

2) a function $\kappa(x, u, t)$ has finite one-side limiting values along boundaries $\Gamma_{i}$,

3) the set consisting of all boundaries $\Gamma_{i}$ has zero measure.

A function $\kappa(x, u, t)$ is said to be piecewise continuous in $H \times R^{m+1}$ if it is piecewise continuous in each finite domain $G \subset H \times R^{m+1}$.

If $u(t) \in R^{m}$ is an absolutely continuous function, then a solution to the equation (1) is 
defined [6] as an absolutely continuous solution to the differential inclusion $\dot{x}(t) \in A x(t)+F(x, t)$, where $F(x, t)$ is a minimum convex closed set containing all limiting values $f\left(x^{*}, u(t), t\right)+$ $\beta\left(x^{*}, u(t), t\right) b\left(x^{*}, u(t), t\right) \dot{u}(t)$ as $x^{*} \rightarrow x, t=$ const, while points $\left(x^{*}, u(t), t\right)$ are not included in a discontinuity set of the function $f(x, u(t), t)+\beta(x, u(t), t) b(x, u(t), t) \dot{u}(t)$.

The existence and uniqueness conditions for an absolutely continuous solution to the equation (1) are given in the next lemma that is a direct corollary to theorem 1 [11].

Lemma: Let the above conditions hold, and the functions $f(x, u, t), \beta(x, u, t) b(x, u, t)$ satisfy the one-sided Lipschitz condition in $x$

$$
\begin{gathered}
(x-y, f(x, u, t)-f(y, u, t)) \leq m_{1}(t, u)(x-y, x-y) \\
\left((\beta(x, u, t) b(x, u, t)-\beta(y, u, t) b(y, u, t))^{*}(x-y)\right) \leq m_{2}(t, u)(x-y, x-y),
\end{gathered}
$$

where functions $m_{1}(t, u) \in R, m_{2}(t, u) \in R^{m}$ are integrable in $t, u ; B^{*}: H \rightarrow R^{m}$ is an operator adjoint to an operator $B: R^{m} \rightarrow H$.

Then there exists a unique absolutely continuous solution to the equation (1) corresponding to an absolutely continuous function $u(t) \in R^{m}$.

In case of an arbitrary non-decrease function $u(t) \in R^{m}$, a solution to the equation (1) is defined as a vibrosolution [9]. A vibrosolution is expected to be a function discontinuous at discontinuity points of the function $u(t)$.

Definition 1: The convergence in the Hilbert space $H$

$$
*-\lim x^{k}(t)=x(t), \quad t \geq t_{0}
$$

is said to be the $*$-weak convergence if the following conditions hold

1) $\quad \lim \left\|x^{k}\left(t_{0}\right)-x\left(t_{0}\right)\right\|=0, t \geq t_{0}$,

2) $\quad \lim \left\|x^{k}(t)-x(t)\right\|=0, t \geq t_{0}$, in all continuity points of the function $x(t)$,

3) $\sup _{k} \operatorname{Var}_{\infty}\left[t_{0}, T\right] x^{k}(t)<\infty$ for any $T \geq t_{0}$, where a variation of a function $x(t) \in H$ is defined by

$$
\operatorname{Var}_{\infty}[a, b] x(t)=\|x(t)\|+\sup _{\tau} \sum_{i=1}^{N}\left\|x\left(t_{i}\right)-x\left(t_{i-1}\right)\right\|,
$$

and supremum is over all possible partitions $\tau=\left(a=t_{0}, t_{1}, \ldots, t_{N}=b\right),\|\cdot\|$ is the norm in the space $H$.

Definition 2: The left-continuous function $x(t)$ is said to be a vibrosolution to the equation (1) if the *-weak convergence of an arbitrary sequence of absolutely continuous non-decreasing functions $u^{k}(t) \in R^{m}$ to a non-decreasing function $u(t) \in R^{m}$

$$
*-\lim u^{k}(t)=u(t)
$$

implies the analogous convergence

$$
*-\lim x^{k}(t)=x(t)
$$

of corresponding solutions $x^{k}(t)$ to the equation

$$
\dot{x}^{k}(t)=A x^{k}(t)+f\left(x^{k}, u^{k}, t\right)+\beta\left(x^{k}, u^{k}, t\right) b\left(x^{k}, u^{k}, t\right) \dot{u}^{k}(t), x^{k}\left(t_{0}\right)=x_{0},
$$


and the unique limit $x(t)$ occurs regardless of a choice of an approximating sequence $\left\{u^{k}(t)\right\}$, $k=1,2, \ldots$

\section{Existence of a Solution. Necessary Conditions}

As in case of a finite-dimensional differential equation in distribution [1,3], existence of a vibrosolution to an equation (1) is closely related to the solvability of a certain associated system in differentials.

Theorem 1: Let the lemma conditions hold.

If a unique vibrosolution to the equation (1) exists, then a system of differential equations in differentials in the space $H$

$$
\frac{d \xi}{d u}=\beta(\xi, u, s) b(\xi, u, s), \quad \xi(\omega)=z
$$

is solvable inside a cone of positive directions $K=\left\{u \in R^{m}: u_{i} \geq \omega_{i}, i=1, \ldots, m\right\}$ with arbitrary initial values $\omega \in R^{m}, \omega \geq u\left(t_{0}\right), z \in H$, and $s \geq t_{0}$.

Proof: Consider a vibrosolution $x(t)$ to an equation (1) with an initial value $x(s)=z$ and a function $u(t)=\omega+(v-\omega) \chi(t-s)$, where $\chi(t-s)$ is a Heaviside function, $\omega, v \in R^{m}, v \geq \omega$, and $s \geq t_{0}$. By virtue of the theorem conditions this vibrosolution exists. Let us prove that under the theorem conditions the Kurzweil equality [10]

$$
x(s+)=y(1)
$$

holds as $x(s+)=\lim x(t), t \rightarrow s+$, where a limit is regarded in the norm of the space $H$, and $y(\tau)$ is a solution to the equation

$$
\frac{d y}{d \tau}=\beta(y, \omega+(v-\omega) \tau, s) b(y, \omega+(v-\omega) \tau, s)(v-\omega), \quad y(0)=z, \quad 0 \leq \tau \leq 1 .
$$

By virtue of the given lemma and the theorem conditions, an absolutely continuous solution to the equation (5) $y(\tau)$ exists and is unique, if $v \geq \omega$.

Following the proof of theorem 1 [12], it is readily verified that under the theorem conditions, the functions $y^{k}(\tau)=x^{k}(s+\tau / k), \quad 0 \leq \tau \leq 1, \quad k=1,2, \ldots$, where $x^{k}(t)$ are vibrosolutions to equations (1) with initial values $x^{k}(s)=z$ and absolutely continuous functions $u^{k}(t)=\omega$, if $t \leq s$, $u^{k}(t)=\omega+k(v-\omega)(t-s)$, if $s \leq t \leq s+1 / k$, and $u^{k}(t)=v$, if $t \geq s+1 / k$, in right-hand sides, are solutions to the equations

$$
\begin{gathered}
\frac{d y^{k}}{d \tau}=\left[A y^{k}(\tau)+f\left(y^{k}, \omega+(v-\omega) \tau, s+\tau / k\right)\right] / k \\
+\beta\left(y^{k}, \omega+(v-\omega) \tau, s+\tau / k\right) b\left(y^{k}, \omega+(v-\omega) \tau, s+\tau / k\right)(v-\omega), y^{k}(0)=z
\end{gathered}
$$

and the following equality holds

$$
x^{k}(s+1 / k)=y^{k}(1)
$$

By virtue of the theorem on continuous dependence of a solution to a differential inclusion on a right-hand side in a Banach space [11], a sequence of absolutely continuous functions $y^{k}(\tau)$ converges to an absolutely continuous solution to the equation (5) pointwise in the norm of the space $H$ :

$$
\lim \left\|y^{k}(\tau)-y(\tau)\right\|=0, \quad k \rightarrow \infty, \quad \tau \in[0,1]
$$


Thus, $\lim y^{k}(1)=y(1), \quad k \rightarrow \infty$, and by virtue of $(6)$

$$
\lim _{t \rightarrow s+} \lim _{k \rightarrow \infty} x^{k}(t)=y(1) .
$$

Taking into account the equality

$$
\lim _{t \rightarrow s+} \lim _{k \rightarrow \infty} x^{k}(t)=x(s+),
$$

where $x(t)$ is a vibrosolution to the equation (1), the Kurzweil equality (4) is proved.

Define the function $\xi(z, \omega, v, s)$ by

$$
\xi(z, \omega, v, s)=y(1)=z+\int_{0}^{1} \beta(y(\tau), \omega+(v-\omega) \tau, s) b(y(\tau), \omega+(v-\omega) \tau, s)(v-\omega) d \tau
$$

where $y(\tau)$ is a solution to the equation (5). Since a solution $y(\tau)$ exists and is unique under the theorem conditions, if $v \geq \omega$, the function $\xi(z, \omega, v, s) \in H$ is uniquely defined inside a cone $K=\left\{u \in R^{m}: u_{i} \geq \omega_{i}, i=1, \ldots, m\right\}$. It only remains to prove that

$$
\frac{d \xi(z, \omega, v, s)}{d v}=\beta(\xi(z, \omega, v, s), v, s) b(\xi(z, \omega, v, s), v, s) .
$$

However, the proof of this correlation is quite consistent with the last part of the proof of theorem 1 [12] and can be omitted here. Thus, the function $\xi(z, \omega, v, s)$ defined by (7) is a unique solution to the system of equations in differentials (3) inside a cone $K$ as $s \geq t_{0}$. Theorem 1 is proved.

\section{Existence of a Solution. Sufficient Conditions}

Let us prove that under additional conditions imposed on a function $\beta(x, u, t) b(x, u, t)$ the necessary existence conditions for a vibrosolution to an equation (1) coincide with the sufficient ones.

Theorem 2: Let 1) the lemma conditions hold, and, moreover,

2) $\{\partial b(x, u, t) / \partial x\} \in L\left(H \rightarrow L\left(R^{m} \rightarrow H\right)\right),\{\partial b(x, u, t) / \partial t\} \in L\left(R^{m} \rightarrow H\right)$ be bounded continuous defined in the space $H \times R^{m} \times R$,

3) functions $\partial \beta(x, u, t) / \partial x, \partial \beta(x, u, t) / \partial t$ be piecewise continuous in $x, u, t$ and their continuity domains be locally connected.

If a system of differential equations in differentials (3) is solvable inside a cone of positive directions $K=\left\{u \in R^{m}: u_{i} \geq \omega_{i}, i=1, \ldots, m\right\}$ with arbitrary initial values $\omega \in R^{m}, \omega \geq u\left(t_{0}\right)$, $z \in H$, and $s \geq t_{0}$, then a unique vibrosolution to the equation (1) exists.

Proof: Let $\left[u^{k}(t)\right\}, k=1,2, \ldots$, be a sequence of absolutely continuous non-decreasing functions $u^{k}(t) \in R^{m}$, which tends to a distribution function $u(t)$ in the sense of the *-weak convergence. Consider the equation (1) with absolutely continuous non-decreasing functions $u^{k}(t)$ in a right-hand side, that is

$$
\dot{x}^{k}(t)=A x^{k}(t)+f\left(x^{k}, u^{k}, t\right)+\beta\left(x^{k}, u^{k}, t\right) b\left(x^{k}, u^{k}, t\right) \dot{u}^{k}(t), \quad x^{k}\left(t_{0}\right)=x_{0} .
$$

It should be noted that the theorem conditions (1)-(3), the lemma of Section 2, and the theorem 1 [11] yield existence and uniqueness of an absolutely continuous solution to the equation (8). As follows from $[7,8]$, this solution can be represented as a Fourier sum in the space $H$ on the com- 
plete orthonormal basis $\left\{c_{i}\right\}_{i=0}^{\infty}$ generated by eigenfunctions of the operator $A$

$$
x^{k}(t)=\sum_{i=0}^{\infty} x_{i}^{k}(t) c_{i}
$$

Scalar functions $x_{i}^{k}(t)$ satisfy the equations

$$
d x_{i}^{k}(t)=\lambda_{i} x_{i}^{k}(t) d t+f_{i}\left(x_{i}^{k}, u^{k}, t\right)+\beta\left(x_{i}^{k}, u^{k}, t\right) b_{i}\left(x_{i}^{k}, u^{k}, t\right) d u^{k}(t), \quad x_{i}^{k}(0)=x_{i 0}
$$

$\left\{\lambda_{i}\right\}_{i=0}^{\infty}$ is a countable set [7] of eigenvalues of the operator $A$, and $f_{i}(x, u, t) \in R$, $b_{i}(x, u, t) \in R^{m}, x_{i 0} \in R, i=0,1,2, \ldots$, are Fourier coefficients for a function $f(x, u, t)$, an operator $b(x, u, t)$, and an initial value $x_{0}$ on the basis $\left\{c_{i}\right\}_{i=0}^{\infty}$, respectively:

$$
f(x, u, t)=\sum_{i=0}^{\infty} f_{i}(x, u, t) c_{i}, \quad b(x, u, t)=\sum_{i=0}^{\infty} b_{i}(x, u, t) c_{i}, \quad x_{0}=\sum_{i=0}^{\infty} x_{i 0} c_{i} .
$$

The convergence of the Fourier series (11) is regarded in the norms of the corresponding Hilbert spaces.

Consider an infinite $(i=0,1,2, \ldots)$ number of finite-dimensional equations (10) which contain an arbitrary non-decreasing function $u(t) \in R^{m}$ in right-hand sides

$$
d x_{i}(t)=\lambda_{i} x_{i}(t) d t+f_{i}\left(x_{i}, u, t\right)+\beta\left(x_{i}, u, t\right) b_{i}\left(x_{i}, u, t\right) d u(t), \quad x_{i}(0)=x_{i 0},
$$

whose solutions are thus regarded as vibrosolutions. Since $x_{i}(t) \in R$ are scalar functions, existence and uniqueness of solutions to the equations (12) are assured of the existence and uniqueness theorem for a vibrosolution [3] by virtue of the inequalities $\operatorname{Re}\left(\lambda_{i}\right)<0$ [7], the theorem conditions (1)-(3), and the solvability of the system of equations in differentials (3) inside a cone $K$. Then, taking into account the vibrosolution definition given in Section 2, we obtain the pointwise convergence of absolutely continuous solutions $x_{i}^{k}(t)$ to the equations $(10)$ to vibrosolutions $x_{i}(t)$ to the equations (12)

$$
\lim \left|x_{i}^{k}(t)-x_{i}(t)\right|=0, \quad k \rightarrow \infty, \quad t \geq t_{0}, \quad i=0,1,2, \ldots,
$$

in all continuity points of the function $u(t)$ as

$$
*-\lim u^{k}(t)=u(t), \quad t \rightarrow \infty,
$$

where $u^{k}(t) \in R^{m}$ are absolutely continuous non-decreasing functions. Thus,

$$
\lim \left\|\sum_{i=0}^{N} x_{i}^{k}(t) c_{i}-\sum_{i=0}^{N} x_{i}(t) c_{i}\right\|=0, \quad k \rightarrow \infty, N<\infty,
$$

in all continuity points of the function $u(t)$.

Consider the Fourier sum generated by the functions $x_{i}(t)$ on the basis $\left\{c_{i}\right\}_{i=0}^{\infty}$

$$
\sum_{i=0}^{\infty} x_{i}(t) c_{i}
$$

Let us prove that the series (13) converges in the norm of the space $H$. Indeed, the following inequalities [5] hold

$$
\begin{aligned}
& \left\|\sum_{i=N}^{\infty} x_{i}(t) c_{i}\right\| \\
& \quad=\left\|\sum_{i=N}^{\infty}\left\{x_{i 0} \exp \left(\lambda_{i}(t)\right)+\int_{t_{0}}^{t} \exp \left(\lambda_{i}(t-s)\right)\left[f_{i}\left(x_{i}, u, s\right) d s+\beta\left(x_{i}, u, s\right) b_{i}\left(x_{i}, u, s\right) d u(s)\right]\right\} c_{i}\right\|
\end{aligned}
$$




$$
\begin{gathered}
\leq \sum_{i=N}^{\infty}\left\|x_{i 0} \exp \left(\operatorname{Re}\left(\lambda_{i}(t)\right)\right)\right\|+\sum_{i=N}^{\infty}\left\|\int_{t_{0}}^{t} \exp \left(\operatorname{Re}\left(\lambda_{i}(t-s)\right)\right) f_{i}\left(x_{i}, u, s\right) d s\right\| \\
\quad+\sum_{i=N}^{\infty}\left\|\int_{t_{0}}^{t} \exp \left(\operatorname{Re}\left(\lambda_{i}(t-s)\right)\right) \beta\left(x_{i}, u, s\right) b_{i}\left(x_{i}, u, s\right) d u(s)\right\|<\infty
\end{gathered}
$$

since functions $f_{i}(x, u, t)$ and $\beta(x, u, t) b_{i}(x, u, t)$ are bounded and satisfy the one-sided Lipschitz condition, $\lim \operatorname{Re}\left(\lambda_{i}\right)=-\infty$ as $i \rightarrow \infty$ [7], $t-s \geq 0$, the Fourier series (11) converge, and the latter integral is with a bounded variation function $u(t)$. Thus, the Fourier series (13) converges, i.e., there exists an $H$-valued function $x(t) \in H$ such that

$$
\lim \left\|x(t)-\sum_{i=0}^{N} x_{i}(t) c_{i}\right\|=0, \quad N \rightarrow \infty .
$$

Let us finally prove that the function $x(t) \in H$ obtained as a Fourier sum (13) is a vibrosolution to the equation (1). For any $\epsilon>0$ there exist a number $N_{1}$ such that the inequality

$$
\left\|x(t)-\sum_{i=0}^{N_{1}} x_{i}(t) c_{i}\right\|<\epsilon / 3
$$

holds by virtue of the convergence of a Fourier series (13), and for any $\epsilon>0$ there exists a number $N_{2}$ such that the inequality

$$
\left\|x^{k}(t)-\sum_{i=0}^{N_{2}} x_{i}^{k}(t) c_{i}\right\|<\epsilon / 3,
$$

holds by virtue of convergence of a Fourier series (9), Moreover, for any $\epsilon>0$ there exist a number $N=\max \left(N_{1}, N_{2}\right)$ and a number $K$ such that for any $k \geq K$ the inequality

$$
\left\|\sum_{i=0}^{N} x_{i}(t) c_{i}-\sum_{i=0}^{N} x_{i}^{k}(t) c_{i}\right\|<\epsilon / 3
$$

holds in all continuity points of the function $u(t)$, since $x_{i}(t)$ is a vibrosolution to the equation (12) and $\left\{x_{i}^{k}(t)\right\}, k=1,2, \ldots$, is a sequence of approximating solutions to the equations (10). Thus, for any $\epsilon>0$ there exist a number $N$ and number $K$ such that for any $k \geq K$ we obtain

$$
\begin{gathered}
\left\|x(t)-x^{k}(t)\right\| \leq\left\|x(t)-\sum_{i=0}^{N} x_{i}(t) c_{i}\right\| \\
+\left\|\sum_{i=0}^{N} x_{i}(t) c_{i}-\sum_{i=0}^{N} x_{i}^{k}(t) c_{i}\right\|+\left\|x^{k}(t)-\sum^{N} x_{i}^{k}(t) c_{i}\right\|<\epsilon,
\end{gathered}
$$

in all continuity points of the function $u(t)$. The inequalities (14) yield the convergence in the norm of the space $H$

$$
\lim \left\|x^{k}(t)-x(t)\right\|=0, \quad k \rightarrow \infty
$$

in all continuity points of the function $u(t)$. Moreover,

$$
x^{k}\left(t_{0}\right)=x\left(t_{0}\right)=x_{0}
$$

by virtue of coincidence of initial values of the equations (1) and (8), and the inequality

$$
\sup \operatorname{Var}_{\infty}\left[t_{0}, t\right] x^{k}(T)<\infty \text { for any } T \geq t_{0}
$$


holds by virtue of the uniform boundedness of variations of absolutely continuous functions $x_{i}^{k}(t)$, $\sup \operatorname{Var}\left[t_{0}, T\right] x_{i}^{k}(t)<\infty$ for all $i=0,1,2, \ldots$ and any $T \geq t_{0}$, and the convergence of the Fourier series (9). Thus, the $*$-weak convergence in the space $H$

$$
*-\lim x^{k}(t)=x(t), \quad k \rightarrow \infty, \quad t \geq t_{0}
$$

is proved. Since $x^{k}(t), k=1,2, \ldots$, are absolutely continuous solutions to the equations (8) that are equations (1) with absolutely continuous non-decreasing functions $u^{k}(t) \in R^{m}$ in right-hand sides, the vibrosolution definition implies that the function $x(t) \in H$ is a vibrosolution to the equation (1). Theorem 2 is proved.

Remark: The vibrosolution definition as well as the necessary and sufficient existence conditions can also be stated for nonmonotonic functions $u(t) \in R^{m}$, assuming that the one-sided Lipschitz condition holds for a function $\beta(x, u, t) b(x, u, t) \operatorname{sign}(\dot{u}(t))$ and approximating functions $\beta\left(x^{k}, u^{k}, t\right) b\left(x^{k}, u^{k}, t\right) \dot{u}^{k}(t)$ for any $k=1,2, \ldots$.

\section{Equivalent Equation with a Measure}

It should be noted that only vibrosolutions, which correspond to absolutely continuous functions $u^{k}(t) \in R^{m}$, are absolutely continuous solutions to a differential equation in distribution (1). Therefore, it is not clear how to compute jumps of a vibrosolution to a differential equation in distribution at discontinuity points of an arbitrary non-decreasing function $u(t) \in R^{m}$. Thus, it is helpful to design an equivalent equation with a measure whose conventional (in the sense of the definition of a solution to an ordinary differential equation with a discontinuous right-hand side that is given in Section 2) solution coincides with a vibrosolution to an equation (1), and which enables us to directly compute jumps of a solution at discontinuity points of an arbitrary non-decreasing function $u(t) \in R^{m}$.

Theorem 3: Let the theorem 2 conditions hold. Then an equation (1) and an equivalent equation with a measure

$$
\begin{gathered}
d y(t)=A y(t) d t+f(y, u, t) d t+\beta(y, u, t) b(u, u, t) d u^{c}(t) \\
+\sum_{t_{i}} G\left(y\left(t_{i}-\right), u\left(t_{i}-\right), \Delta u\left(t_{i}\right), t_{i}\right) d \chi\left(t-t_{i}\right), \quad y\left(t_{0}\right)=x_{0} .
\end{gathered}
$$

have the same unique solution regarded for in equation (1) as a vibrosolution.

Here $G(z, v, u, s)=\xi(z, v, v+u, s)-z$, where $\xi(z, v, u, s)$ is a solution to a system of equations in differentials $(3) ; u^{c}(t)$ is a continuous component of a non-decreasing function $u(t)$, $\Delta u\left(t_{i}\right)=u\left(t_{i}+\right)-u\left(t_{i}-\right)$ is a jump of a function $u(t)$ at $t_{i}, t_{i}$ are discontinuity points of a function $u(t), \chi\left(t-t_{i}\right)$ is a Heaviside function.

Proof: A function of jumps $G(z, \omega, u, s)$ is bounded in the norm of the space $H$ as a solution to the system (3) with a right-hand side $\beta(\xi, u, s) b(\xi, u, s)$ satisfying the one-sided Lipschitz condition. Then, by virtue of the lemma of Section 2 and the theorem 1 [11], a solution to the equation with a measure (15) exists and is unique as a bounded variation function with an absolutely continuous component in continuity intervals of the function $u(t)$ and the jumps determined by the function $G\left(y\left(t_{i}-\right), u\left(t_{i}-\right), \Delta u\left(t_{i}\right), t_{i}\right)$ at discontinuity points of the function $u(t)$. As follows from $[7,8]$, this solution can be represented as a Fourier sum in the space $H$ on the complete orthonormal basis $\left\{c_{i}\right\}_{i=0}^{\infty}$ generated by eigenfunctions of the operator $A$ :

$$
\sum_{i=0}^{\infty} y_{i}(t) c_{i}
$$


Scalar functions $y_{i}(t)$ satisfy the equation

$$
\begin{gathered}
y_{i}(t)=\lambda_{i} y_{i}(t) d t+f_{i}\left(y_{i}, u, t\right)+\beta\left(y_{i}, u, t\right) b_{i}\left(y_{i}, u, t\right) d u^{c}(t) \\
+\sum_{t_{i}} G_{i}\left(y_{i}\left(t_{i}-\right), u\left(t_{i}-\right), \Delta u\left(t_{i}\right), t_{i}\right) d \chi\left(t-t_{i}\right), \quad y_{i}\left(t_{0}\right)=x_{i 0}
\end{gathered}
$$

where scalar functions $G_{i}\left(z_{i}, \omega, u, s\right)$ are defined as follows

$$
G_{i}\left(z_{i}, \omega, u, s\right)=\xi_{i}\left(z_{i}, \omega, \omega+u, s\right)-z_{i}
$$

and functions $\xi_{i}\left(z_{i}, \omega, u, s\right), u \geq \omega, s \geq t_{0}$, are solutions to the equations in differentials

$$
\frac{d \xi_{i}}{d u}=\beta\left(\xi_{i}, u, s\right) b_{i}\left(\xi_{i}, u, s\right), \quad \xi_{i}(\omega)=z_{i}, \quad i=0,1,2, \ldots
$$

inside cones of positive directions $K=\left\{u \in R^{m}: u_{j} \geq \omega_{j}, j=1, \ldots, m\right\}$ with arbitrary initial values $\omega \in R^{m}, z_{i} \in R$, and $s \geq t_{0}$.

Consider an equation (1) with an arbitrary non-decreasing function $u(t) \in R^{m}$ in a right-hand side. Existence and uniqueness of a vibrosolution to such an equation have already been proved in the theorem 2. That vibrosolution can also be represented as a Fourier sum (13) on the basis $\left\{c_{i}\right\}_{i=0}^{\infty}$ :

$$
x(t)=\sum_{i=0}^{\infty} x_{i}(t) c_{i}
$$

where scalar functions $x_{i}(t)$ satisfy the equations (12)

$$
d x_{i}(t)=\lambda_{i} x_{i}(t) d t+f_{i}\left(x_{i}, u, t\right)+\beta\left(x_{i}, u, t\right) b_{i}\left(x_{i}, u, t\right) d u(t), \quad x_{i}(0)=x_{i 0} .
$$

The equations (12) and (17) are finite-dimensional equations whose right-hand sides contain vector distribution and piecewise continuous regular functions satisfying the one-sided Lipschitz condition. Thus, by the virtue of theorem 2 [3], unique solutions $x_{i}(t)$ and $y_{i}(t)$ to the equations (12) and (17) coincide as vibrosolutions for any $i=0,1,2, \ldots$. The vibrosolution definition given in Section 2 implies that for any $i=0,1,2 \ldots$, the equalities

$$
\left|x_{i}(t)-y_{i}(t)\right|=0, \quad t \geq t_{0}
$$

hold in all continuity points of the function $u(t)$.

Let us finally prove that solutions to the equations (1) and (15) are indistinguishable as vibrosolutions. Let

$$
\|x(t)-y(t)\| \neq 0, \quad t \geq t_{0}
$$

for at least one continuity point of the function $u(t) \in R^{m}$. Expand the mentioned solutions into Fourier series on the basis $\left\{c_{i}\right\}_{i=0}^{\infty}$ :

$$
x(t)-y(t)=\sum_{i=0}^{\infty}\left(x_{i}(t)-y_{i}(t)\right) c_{i}, \quad t \geq t_{0} .
$$

By virtue of the equalities (18) and the uniqueness of the expansion into a Fourier series on the given basis, the equalities

$$
\|x(t)-y(t)\| \leq \sum_{i=0}^{\infty}\left|x_{i}(t)-y_{i}(t)\right|=0, \quad t \geq t_{0}
$$

also hold in all continuity points of the function $u(t)$. Thus, the vibrosolution definition given in 
Section 2 implies that the functions $x(t) \in H$ and $y(t) \in H$ are indistinguishable as vibrosolutions to the equations (1) and (15). In other words, the equations (1) and (15) have the same unique vibrosolution. Theorem 3 is proved.

\section{Conclusion}

The vibrosolution definition assumes uniqueness of a vibrosolution to an equation (1). This enables us to apply the obtained sufficient existence conditions for a vibrosolution to filtering equations for an infinite-dimensional process over discontinuous observations, for example [13], in case of simultaneous impulses in all observation channels (a scalar function $u(t)$ ). However, the results of this paper also enable us to consider a case of non-simultaneous impulses in observation channels (a vector function $u(t)$ ).

\section{References}

[1] Basin, M.V. and Orlov, Yu.V., Guaranteed estimation of a state of linear dynamic systems over discrete-continuous observations, Automation and Remote Control 53:3 (1992), 349-357.

[2] Basin, M.V., On ellipsoidal estimation of an infinite-dimensional process under uncertainty conditions, Preprints of the 10th IFAC Symposium on System Identification, Copenhagen 1 (1994), 209-214.

[3] Basin, M.V., On a unique solution to a differential equation in vector distribution (Abstract), Journal of Applied Math. and Stoch. Analysis 8:2 (1995), 589-590.

[4] Bressan, A. and Rampazzo, F., On differential systems with quadratic impulses and their applications to Lagrangian mechanics, SIAM J. on Control and Optim. 31:5 (1993), 12051220.

[5] Curtain, R.F. and Pritchard, A.J., Infinite dimensional linear systems theory, Lect. Notes in Control and Info Sci. 8 (1978), Springer-Verlag, New York.

[6] Filippov, A.F., Differential Equations with Discontinuous Right-Hand Sides, Kluwer, New York 1988.

[7] Henry, D., Geometric theory of semilinear parabolic equations, Lect. Notes in Math. 840 (1981), Springer-Verlag Berlin.

[8] Krasnosel'skii, M.A., Zabreiko, P.P., Pustylnik, E.I. and Sobolevskii, P.E., Integral Operators in Spaces of Summarized Functions (in Russian), Nauka, Moscow 1966.

[9] Krasnosel'skii, M.A. and Pokrovskii, A.V., Systems with Hysteresis, Springer-Verlag, New York 1989.

[10] Kurzweil, J., Generalized ordinary differential equations, Czechosl. Math. J. 8:3 (1958), $360-388$.

[11] Levakov, A.A., Certain properties of solutions to differential equations in a Banach space (in Russian), Vestnik of the Byelorussian State Univ. Ser. 1: Physics, Mathematics, Mechanics 2 (1981), 54-56.

[12] Orlov, Yu.V., Vibrocorrect differential equations with a measure, Math. Notes 38:1 (1985), 110-119.

[13] Orlov, Yu.V. and Basin, M.V., On filtering of the Hilbert space-valued stochastic process over discrete-continuous observations, Lecture Notes in Control and Info Sci. 185, Springer-Verlag (1993), 336-346.

[14] Ovseevich, A.I., Trushchenkov, V.L. and Chernousko, F.L., Equations of continuous guaranteed state estimation for dynamical systems, Izvestiya of the USSR Acad. of Sci. Eng. Cybernetics 4 (1984), 94-101. 


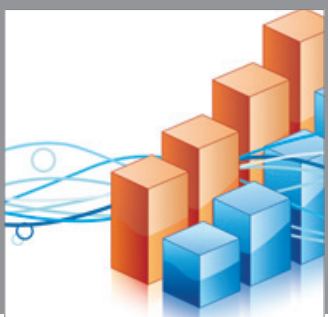

Advances in

Operations Research

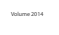

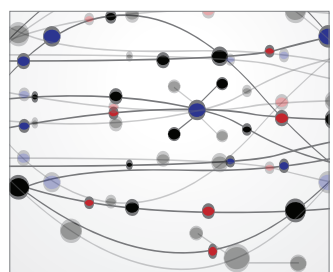

\section{The Scientific} World Journal
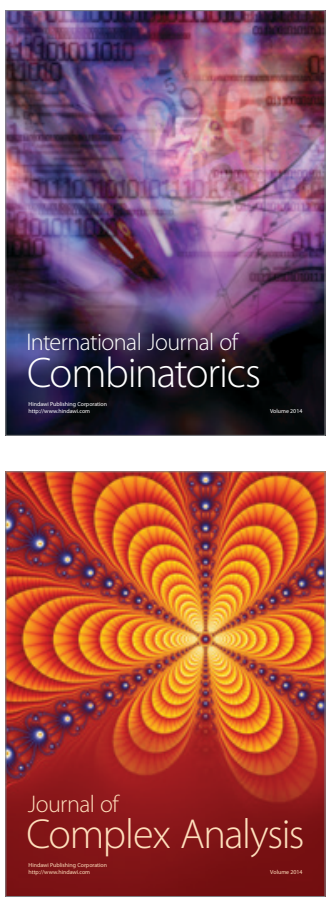

International Journal of

Mathematics and

Mathematical

Sciences
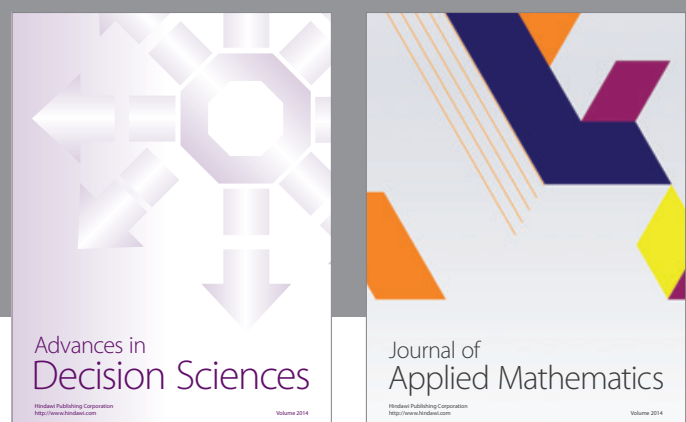

Journal of

Applied Mathematics
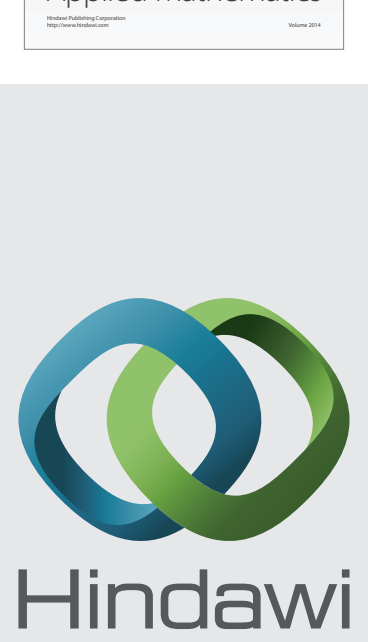

Submit your manuscripts at http://www.hindawi.com
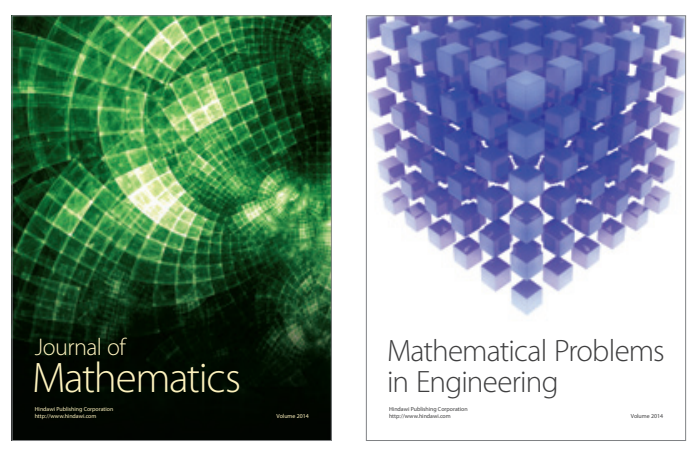

Mathematical Problems in Engineering
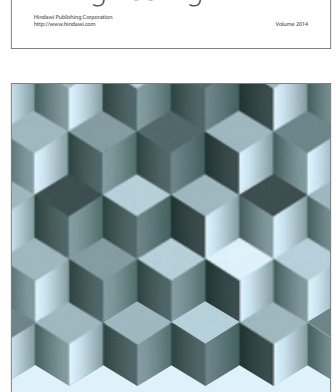

Journal of

Function Spaces
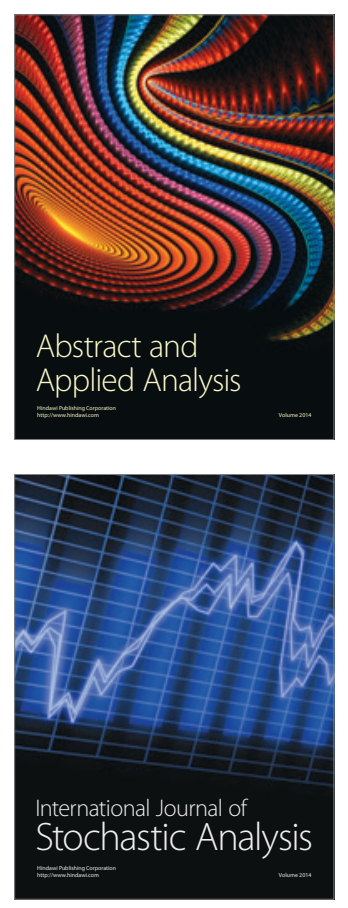

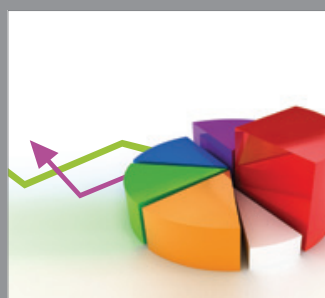

ournal of

Probability and Statistics

Promensencen
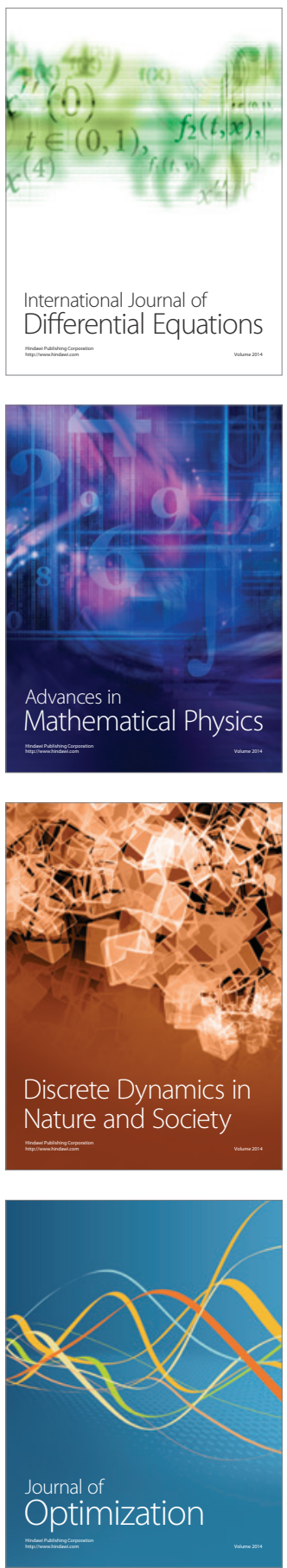